\title{
Snap-Action Bistable Micromechanisms Actuated by Nonlinear Resonance
}

\author{
Jasmina Casals-Terré, Andreu Fargas-Marques, and Andrei M. Shkel, Associate Member, IEEE, Member, ASME
}

\begin{abstract}
This paper presents analysis, design, realization, and experimental demonstration of a bistable switch actuated dynamically utilizing mechanical resonance phenomenon. We demonstrated that if a bistable structure is driven into a resonance near one of its states, it may achieve a large enough amplitude of vibration, sufficient to switch between its stable states. Using energy analysis, we concluded that dynamic switching of bistable structures may provide significant energy advantages over conventional static-switching approaches. To confirm the results, we derived analytically the closed-form actuation conditions guaranteeing switching between the states of a bistable structure and applied these conditions to experimental devices. Micromachined prototypes of dynamically actuated bistable switches were designed, fabricated, and characterized. We demonstrated experimentally that resonant dynamic switching provides energy saving of around $60 \%$ at atmospheric pressure with proportional increase in efficiency as the pressure decreases.

[2008-0057]
\end{abstract}

Index Terms-Bistability, dynamic snapping, nonlinear resonance.

\section{INTRODUCTION}

B ISTABILITY is a nonlinear phenomena often used to define precisely two statically stable states. This method is often used due to its power efficiency, as no power is required to keep the structure in its bistable state. Conventionally, the switching between bistable states is static, utilizing either electrostatic [1] or thermal actuation [2].

Bistable microdevices take advantage of bistability, to save energy specifically in applications when the structure needs to be kept in one of the states for a prolonged period of time. Some of these explored mechanisms are latch-lock mechanisms [3], hinged multisegment mechanisms [4]-[6], and the residualcompressive-stress buckled-beam mechanisms [7], [8].

Bistable devices can be actuated using different switching strategies: static or dynamic. Static switching can be achieved when a critical and large force is applied to the mechanism, causing snapping to a second stable state. If the force is applied at a certain frequency, the switching to the second stable state

Manuscript received March 6, 2008; revised June 26, 2008. First published September 5, 2008; current version published October 1, 2008. Subject Editor H. Fujita.

J. Casals-Terre is with the Department of Mechanical Engineering, Technical University of Catalonia, 08222 Terrassa, Spain (e-mail: Jasmina.casals@ upc.edu).

A. Fargas-Marques is with the Institute of Industrial and Control Engineering, Technical University of Catalonia, 08028 Barcelona, Spain (e-mail: Andreu.Fargas@upc.edu).

A. M. Shkel is with the Department of Mechanical Engineering, University of California, Irvine, CA 92697-3975 USA (e-mail: ashkel@uci.edu).

Color versions of one or more of the figures in this paper are available online at http://ieeexplore.iee.org.

Digital Object Identifier 10.1109/JMEMS.2008.2003054 can be achieved at a lower force. For the first time, this phenomenon is explored in this paper.

The micromechanism reported in this paper illustrates an energy-efficient approach for dynamic switching between states of a bistable mechanism. The efficiency is achieved by driving the structure in resonance in the neighborhood of a bistable state; see Fig. 1. When due to resonance the large amplitude is achieved, the structure escapes from a statically stable state and jumps to the second stable state. The actuation principle analyzed in this paper utilizes electrostatic actuation to drive the microstructure into oscillation. This oscillation is amplified at the nonlinear resonance frequency of the structure, achieving a large deviation from its static equilibrium sufficient to switch between two stable states of a bistable structure. Utilization of this switching strategy has been previously employed to actuate monostable microstructures and analytically demonstrated that less power consumption is required as compared to the static case [9]. The switching concept can be applied to any type of portable devices such as displays [10], threshold accelerometers [11], or switches in wireless RF circuits [12]-[14].

This paper investigates the problem of nonlinear resonance of bistable mechanisms and derives the combination of voltages to achieve dynamic snapping. This paper is an extension of previously published results by this group [15].

The rest of this paper is organized as follows. In Section II, an analytical model of a double built-in prebuckled beam is derived. Using this model and incorporating the electrostatic driving force, we derived in Section III the static, dynamic, and the nonlinear resonance snapping voltages. Section IV presents experimental results obtained from Silicon-on-Insulator (SOI) bulk-micromachined prototypes and validates the analytically predicted response. This paper is concluded by summarizing results in Section V.

\section{Analytical Model}

Euler-Bernouilli theory of thin beams is used to model the behavior of the built-in prebuckled beam [16], [17]. When a beam oscillates without dissipation of energy, the total energy of the system is a sum of potential energy stored in the beam due to deformation and kinetic energy of motion. The change in kinetic energy of a differential element of the beam is given by

$$
d U_{k}=\frac{1}{2} m\left(\frac{\partial w}{\partial t}\right)^{2} d x
$$

where $m$ is the mass per unit length $d x$ of the undeformed beam and $w$ is the total deflection of a clamped-clamped 


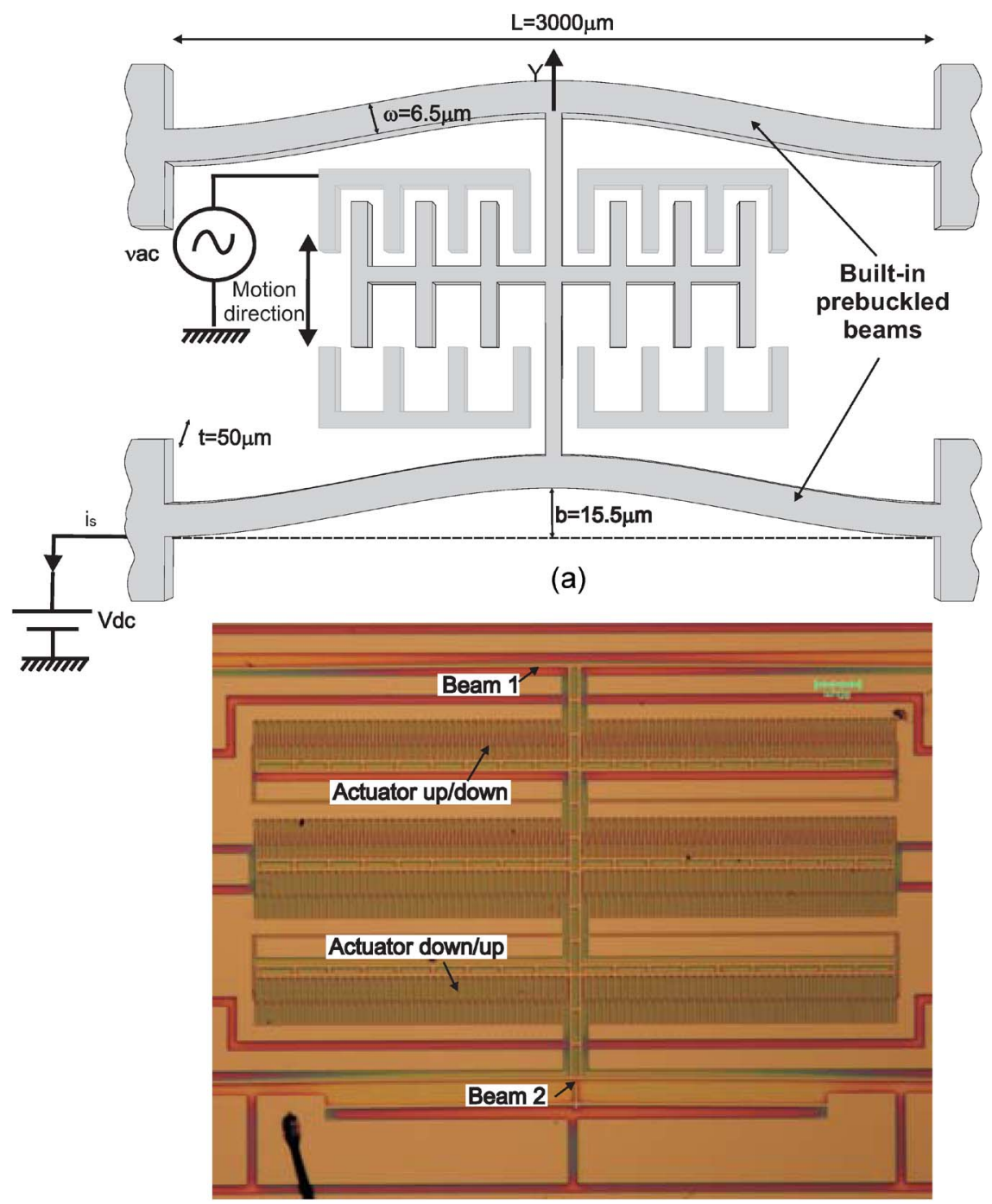

(b)

Fig. 1. (a) Schematics of a bistable mechanism used to achieve dynamic switching between bistable states. (b) Photograph of a bistable micromechanism prototype utilizing the built-in prebuckled double-beam structure.

prebuckled beam. Integrating (1) over the length of the beam yields the kinetic energy expression

$$
U_{k}=\frac{1}{2} m \int_{0}^{L}\left(\frac{\partial w}{\partial t}\right)^{2} d x .
$$

Considering Euler-Bernouilli assumptions in the case of a clamped-clamped built-in prebuckled beam, the potential energy of the built-in prebuckled beam is

$$
U_{p}=V_{a}+V_{b}+V_{c}
$$

where $V_{a}$ is the potential energy due to bending, $V_{b}$ is the potential energy due to axial loads, and $V_{c}$ is the potential energy due to midplane stretching. The induced axial force due to the midplane stretching can be expressed as

$$
S=\frac{E A}{2 L} \int_{0}^{L}\left(\frac{\partial w}{\partial x}\right)^{2} d x
$$

where $E A / L$ is the axial stiffness of the beam; $E$ is the Young modulus of the material, $A$ is the cross section of the beam, and $L$ is the length of the beam. Subsequently, the total compressive force acting on the beam is given by

$$
N=P-\frac{E A}{2 L} \int_{0}^{L}\left(\frac{\partial w}{\partial x}\right)^{2} d x
$$

where $P$ is the initial compressive load applied to the beam. The compressive load can be expressed as

$$
P=P_{\mathrm{cr}}+\frac{E A}{2 L} \int_{0}^{L}\left(\frac{\partial w_{0}}{\partial x}\right)^{2} d x .
$$

In this paper, the beam is already buckled, so there is no need to apply the critical force to buckle the beam $\left(P_{\mathrm{cr}}=0\right)$. 
The potential energy due to bending is proportional to the curvature of the beam and is given by

$$
V_{a}=\frac{1}{2} E I \int_{0}^{L}\left(\frac{\partial^{2} v}{\partial x^{2}}\right)^{2} d x .
$$

The potential energy due to the axial force $P$ can be expressed as

$$
V_{b}=-\frac{1}{2} P \int_{0}^{L}\left(\frac{\partial w}{\partial x}\right)^{2} d x .
$$

Finally, the potential energy due to the midplane stretching is given by

$$
V_{c}=\frac{E A}{8 L}\left[\int_{0}^{L}\left(\frac{\partial w}{\partial x}\right)^{2} d x\right]^{2} .
$$

The total potential energy due to deformation of the beam now can be written as [18]

$$
\begin{aligned}
U_{p}=\frac{1}{2} E I \int_{0}^{L}\left(\frac{\partial^{2} v}{\partial x^{2}}\right)^{2} d x & -\frac{1}{2} P \int_{0}^{L}\left(\frac{\partial w}{\partial x}\right)^{2} d x \\
& +\frac{E A}{8 L}\left[\int_{0}^{L}\left(\frac{\partial w}{\partial x}\right)^{2} d x\right]^{2} .
\end{aligned}
$$

These expressions can be evaluated numerically; however, the formulation is usually simplified when analyzing the dynamics of the mechanism using the Galerkin method. This method has been proved to be reliable to model nonlinear behavior of buckled beams [19], [20]. According to the multimode Galerkin discretization method, trial functions can be the linearvibration mode shapes of the buckled beam, which are members of a complete set of functions. Using these assumptions, the dynamic response around the buckled configuration can be represented as

$$
v(x, t)=\sum_{n=1}^{N} \phi_{n}(x) q_{n}
$$

where $N$ is the number of retained modes of interest, the $\phi_{n}(x)$ are the position-dependent mode shapes of the buckled beam, and the $q_{n}(t)$ are generalized coordinates or time-dependent modal displacements. Using one mode approximation, the total response around first buckling mode shape, Fig. 2 can be determined as

$$
w(x, t)=w_{0}+v(x, t)
$$

where $w_{0}$ is the initial shape $\left(w_{0}=b \psi(x)\right), b$ is the initial height at $x=L / 2, \psi(x)$ is the first buckling mode shape, and $v(x, t)$ is the dynamic response around the buckled state. Substituting the dynamic response as a function of the first buckling

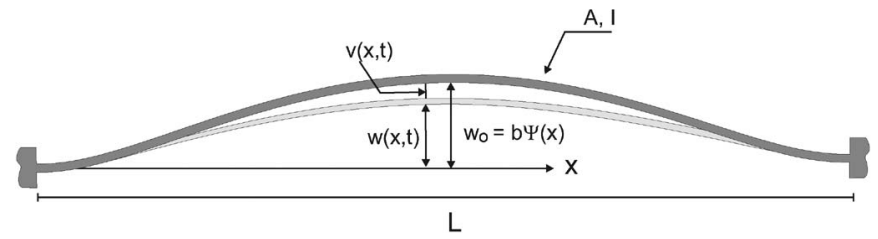

Fig. 2. Scheme of a clamped-clamped built-in prebuckled beam.

mode shape into (12), the total deflection of a clamped-clamped prebuckled beam is described by $\omega(x, t)$

$$
w(x, t)=b \psi(x)+\psi(x) y(t)
$$

where $\psi(x)$ is the first buckling mode shape, see [21], $b$ is the initial apex of the beam, and $y(t)=q(t)$ is the generalized coordinate, in this case, the vertical position of the central point of the beam.

\section{A. Governing Equation of the Built-In Prebuckled Beam}

If the deflection of the beam described by (13) is substituted into (10), the total potential energy of the system can be expressed as

$$
\begin{aligned}
U_{p}= & V_{a}+V_{b}+V_{c} \\
= & \frac{1}{2} E I \int_{0}^{L}\left(\frac{\partial^{2} v(x, t)}{\partial x^{2}}\right)^{2} d x-\frac{1}{2} P \int_{0}^{L}\left(\frac{\partial w(x, t)}{\partial x}\right)^{2} d x \\
& +\frac{E A}{8 L}\left[\int_{0}^{L}\left(\frac{\partial w(x, t)}{\partial x}\right)^{2} d x\right]^{2}
\end{aligned}
$$

where $P$ in the case of a built-in prebuckled beam is

$$
P=\frac{E A}{2 L} \int_{0}^{L}\left(\frac{\partial b \psi(x)}{\partial x}\right)^{2} d x .
$$

Evaluating expression (14), the total potential energy of the system is completely described by the time-position of the beam and its geometric properties

$$
\begin{aligned}
U_{p}= & \frac{1}{32} \frac{E A \pi^{4}}{L^{3}} y(t)^{4}+\frac{1}{8} \frac{E A \pi^{4} b}{L^{3}} y(t)^{3} \\
& +\left(\frac{E I \pi^{4}}{L^{3}}+\frac{1}{8} \frac{E A \pi^{4} b^{2}}{L^{3}}\right) y(t)^{2}-\frac{1}{32} \frac{E A \pi^{4} b^{4}}{L^{3}} .
\end{aligned}
$$

Considering the force as a function of mode coordinate position $y(t)$, the mechanical restoring force of a built-in prebuckled beam can be calculated from (16) by taking the partial derivative of energy with respect to displacement $y$

$$
F_{m}=\frac{\partial U_{p}}{\partial y}
$$

The partial derivative provides the equivalent stiffness $k_{\text {eff }}$ of the system

$$
F_{m}=k y(t)+k_{2} y(t)^{2}+k_{3} y(t)^{3}
$$


where

$$
\begin{aligned}
k & =2\left(\frac{E I \pi^{4}}{L^{3}}+\frac{1}{8} \frac{E A \pi^{4} b^{2}}{L^{3}}\right) \\
k_{2} & =\frac{3}{8} \frac{E A \pi^{4} b}{L^{3}} \\
k_{3} & =\frac{1}{8} \frac{E A \pi^{4}}{L^{3}} .
\end{aligned}
$$

Equation (18) describes the force displacement characteristic of a single built-in prebuckled beam.

Using the same approach, an equivalent mass for the mode of oscillation can be obtained, i.e.,

$$
M_{\mathrm{eq}}=\rho A \int_{0}^{L} \psi(x)^{2} d x+m_{p i} \psi\left(x_{p i}\right)^{2}
$$

where $A$ is the cross section of the beam, $\rho$ is the density of the material, $\psi(x)$ is the first buckling mode shape, $m_{p i}$ is the mass of each finger, and $x_{p i}$ is the position of the geometric center.

If sufficiently large force is applied, the midpoint of the beam will displace from a static equilibrium and switch to the second stable state. During transition, the beam adopts a twisting first mode, which is incompatible with the alignment of the electrostatic comb-drive actuation. However, if a single beam is replaced by two coupled beams, the twisting modes of each beam will cancel each other, see Fig. 1. The force needed to achieve switching in a bistable double beam is exactly the double of a single beam; the torsional modes are suppressed, providing a perfect alignment between actuation combs during the actuation.

The model of stiffness obtained in (18) captures inherent characteristics of the static response of the built-in prebuckled beam. However, if the beam is driven dynamically into resonance, damping force and inertial forces need to be considered. If, for example, the electrostatic forces are used, electrostatic potential energy due to comb-drive actuators is

$$
U_{e}=\frac{V^{2}}{2}\left(C_{0}+C_{0} \frac{y}{y_{\mathrm{ov}}}\right)
$$

where $V$ is the voltage applied between combs, $y_{\text {ov }}$ is the overlap between combs, and $C_{0}$ is the nominal comb capacitance

$$
C_{0}=\frac{N \varepsilon_{0} z_{0} y_{\mathrm{ov}}}{g_{0}} .
$$

In this equation, $\varepsilon_{0}$ is the dielectric permitivity, $z_{0}$ is the thickness of the combs, $N$ is the number of combs, and $g_{0}$ is the gap between combs [22]. The resulting electrostatic force applied to the mechanism is $F e=\partial U e / \partial y(t)$.

The damping force present in the system is considered proportional to velocity, $F=-B \dot{y}(t)$. Due to the number of phenomena that affects the damping in the system (viscous, material/intrinsic damping), the damping coefficient will be obtained from the experimental quality factor measured. As the quality factor will be experimentally measured, the resulting value will include, besides the dominant damping due to viscous damping, other damping phenomena which are difficult to analytically calculate.
Then, the governing equation of the built-in prebuckled beam oscillation can be described by a 1-DOF nonlinear model as

$$
M_{\mathrm{eq}} \ddot{y}(t)+B \dot{y}(t)+k y(t)+k_{2} y(t)^{2}+k_{3} y(t)^{3}=F_{e}
$$

where $M_{\mathrm{eq}}, B$, and $k, k_{2}, k_{3}$ are the constant lumped dynamic parameters of mass, damping, and stiffness, respectively, $y$ is the amplitude of oscillation of the central point of the beam, and $F_{e}$ is the electric force generated by the comb-drive set of electrodes.

\section{Snapping Conditions of a Bistable Mechanism}

Equation (23) describes the simplified lumped mass-spring system model of a bistable mechanism which is a nonlinear system. The response of bistable mechanisms is described by a nonlinear relationship between the force applied and the deflection achieved, see Fig. 4. As the external force increases, the deflection increases until it reaches a critical point where the structural stiffness starts to decrease and the system by itself snaps to the second stable state. This becomes more complicated when the system is driven dynamically. To understand the phenomena, we consider the energy of the electromechanical system. The total energy $U_{T}$ can be defined as

$$
U_{T}=U_{k}+U_{p}+U_{e}
$$

where $U_{k}$ is the kinetic energy, $U_{p}$ is the potential energy stored by springs in result of deformation, and $U_{e}$ is the potential energy due to the actuators. The analysis of the energy evolution can be used to determine the snapping conditions of the system, as well as the snapping position or statically stable point.

This section analyzes the snapping phenomena of bistable structures actuated electrostatically under different conditions. The static snapping allows to determine the critical force (or voltage) and the minimum deflection from the static equilibrium for switching between the bistable states. We first introduce the dynamics of the system without considering energy dissipation.

\section{A. Static Snapping}

When the voltage is applied slowly to the system, quasistatic forcing assumptions can be used. It can be assumed that $\ddot{y}=\dot{y}=0$, and the energy of the system is only defined by the potential energy

$$
U=\frac{1}{2} k y^{2}+\frac{1}{3} k_{2} y^{3}+\frac{1}{4} k_{3} y^{4}+\frac{V^{2}}{2}\left(C_{0}+C_{0} \frac{y}{y_{\text {ov }}}\right) .
$$

If the mechanical restoring force is plotted versus electrostatic force (Fig. 4), the system has three equilibrium states: two stable states (initial and final) and one unstable state (transition). If the electrostatic force is lower than the critical force needed to snap to the second stable state, the system can be deflected until a certain static position is reached, such as in case of Fig. 3. However, if the electrostatic force is larger than the static critical point, the system will pass the unstable state and snap to the second stable state (Fig. 4). 


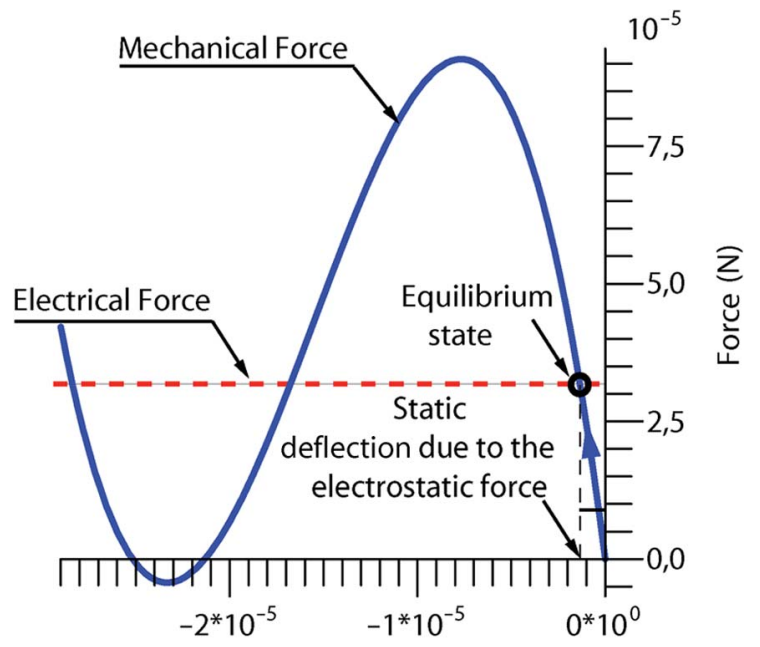

Position of the central point of the beam $(\mathrm{m})$

Fig. 3. If the applied electrostatic force is smaller than the critical mechanical restoring force, the beam deflects until the equilibrium between the mechanical and the electrostatic force is reached.

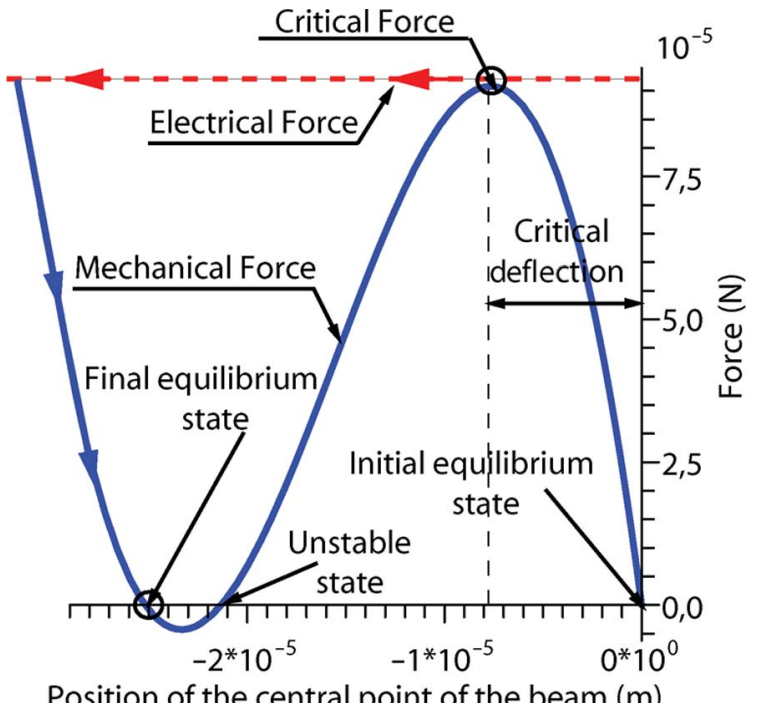

Fig. 4. If the applied electrostatic force (dashed) is larger than the critical restoring mechanical force (continuous), built-in prebuckled beam overcomes the critical deflection and switches to the second stable state (or equilibrium point).

The equilibrium states are those where the variation of energy is zero; this translates to equilibrium between the electrostatic force and the mechanical restoring force of the structure. The effective potential force of the system is

$$
F=\frac{d U}{d y}=k y+k_{2} y^{2}+k_{3} y^{3}+\frac{V^{2}}{2}\left(C_{0} \frac{1}{y_{\mathrm{ov}}}\right) .
$$

The type of equilibrium point is defined by the second derivative of energy or the first derivative of the force

$$
\frac{d F}{d y}=\frac{d^{2} U}{d y^{2}}=k+2 k_{2} y+3 k_{3} y^{2}=0 .
$$

In order to be able to transition from the first stable state to the second, the electrostatic force has to be able to overcome the maximum restoring static force. The deviation that causes
Effective potential force with increasing Vdc $10^{-5}$

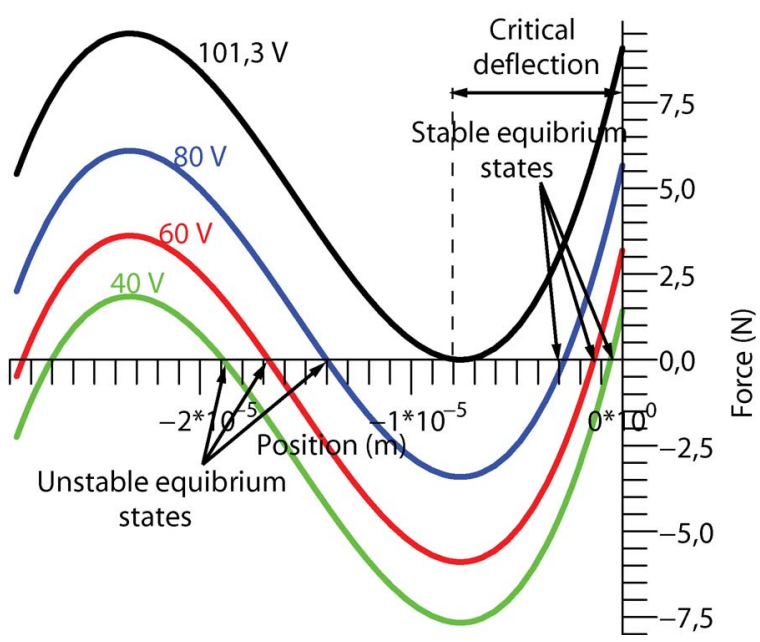

Fig. 5. Evolution of the force profile of a double built-in prebuckled beam, when changing the voltage. When the static snapping voltage $\left(V_{\mathrm{ss}}=101.3 \mathrm{~V}\right)$ is applied, the electrostatic force reaches directly the critical deflection because the mechanical restoring force is completely compensated by the electrostatic force and the system deflects to the second stable state.

the maximum restoring force provided by the mechanism is obtained from (27), where the limit condition for existence of a maximum of the force $d^{2} U / d y^{2}=0$ is satisfied. If this condition is satisfied, and the third derivative of the energy is positive, the force has a maximum. Substituting the calculated value of deviation into (26), the static voltage required to achieve snapping can be obtained. The electrical force and the mechanical restoring spring force compensate each other in this point.

The equilibrium condition (27) provides an analytical value for the maximum displacement before the snapping and the voltage needed to statically reach this point is achieved

$$
\begin{aligned}
y_{\max }= & \frac{-1}{3} \frac{k_{2}-\sqrt{k_{2}^{2}-3 k_{3} k}}{k_{3}} \\
V_{\mathrm{ss}}= & \frac{1}{9} \frac{\sqrt{6 N \varepsilon_{0} z_{0} g_{0}\left(-2 k_{2}^{3}+2 k_{2}^{2} \sqrt{k_{2}^{2}-3 k_{3} k}\right.}}{N \varepsilon_{0} z_{0} k 3} \\
& +\frac{1}{9} \frac{\sqrt{\left.-6 k k_{3} \sqrt{k_{2}^{2}-3 k_{3} k}+9 k k_{3} k_{2}\right)}}{N \varepsilon_{0} z_{0} k_{3}} .
\end{aligned}
$$

Initially, the resulting position of the central point of the beam has three equilibrium points (two stable and one unstable). The increase in the applied electrostatic force to the double built-in prebuckled beam modifies the position where the mechanical restoring force compensates the external electrostatic force and the first stable state approached to the unstable state, see Fig. 5. The voltage is increased until both points merge at the critical point.

\section{B. Dynamic Snapping Due to Step-Function Forcing}

In this section, we consider the case when the bistable mechanism is electrostatically actuated using a step function. Under these forcing conditions, the dynamics of transient response is of interest. For vacuum-packaged devices, the critical 
deflection can be reached by relatively low voltages due to the gained kinetic energy, causing the bistable mechanism to overshoot the critical point. After passing the critical point, the bistable mechanism will transition to the second stable state. This behavior can be explained analytically, from the equation of energy. The evolution of energy over time is defined by

$$
\frac{d U}{d t}=\left(M_{\mathrm{eq}} \ddot{y}+k y+k_{2} y^{2}+k_{3} y^{3}+\frac{V^{2}}{2}\left(C_{0} \frac{1}{y_{\mathrm{ov}}}\right)\right) \dot{y} .
$$

The governing equation of motion of the system is

$$
M_{\mathrm{eq}} \ddot{y}+k y+k_{2} y^{2}+k_{3} y^{3}+\frac{V^{2}}{2}\left(C_{0} \frac{1}{y_{\mathrm{ov}}}\right)=-B \dot{y} .
$$

From (30) and (31), the energy decreases continuously due to damping, until the system reaches a stable state. Therefore, the evolution of the energy is defined by

$$
\frac{d U}{d t}=-B \dot{y}^{2} .
$$

When the suspension of the mechanism deforms, the energy changes and the energy evolution can be determined by

$$
\frac{d U}{d y}=k y+k_{2} y^{2}+k_{3} y^{3}+\frac{V^{2}}{2}\left(C_{0} \frac{1}{y_{\mathrm{ov}}}\right)=0 .
$$

Including dynamics of the system, the slope of energy loss is defined by inertia and damping

$$
\frac{d U}{d y}=-B \dot{y}-M_{\mathrm{eq}} \ddot{y} .
$$

The dynamic snapping voltage is defined as the minimum constant voltage which, when applied in the form of a step function, will allow to pass the critical point to the second stable state. In the dynamic case, voltages lower than dynamic snapping voltage cannot result in snapping. As it has been observed in the previous section, the voltage applied using the comb drives modifies the response of the system. If the voltage is increased, at some point the overshoot could overpass the critical deflection and causes snapping.

Assuming that the energy of the system remains constant, no energy dissipation, after applying ac actuation voltage, the system will deflect until it overshoots the unstable point, and the system will snap automatically to the second stable state of the bistable mechanism. This voltage is called the dynamic snapping voltage $V_{\mathrm{sd}}$. This type of analysis has been previously reported to be suitable for analysis of nonlinear microstructures driven by parallel plates [15]. Considering that the potential energy at the initial position $\left(U_{0}\right)$ is equal to the potential energy of the unstable point $\left(U_{\text {unstable }}\right)$

$$
\begin{aligned}
U_{0} & =\frac{1}{2} C_{0} V_{\mathrm{sd}}^{2} \\
U_{\text {unstable }} & =\frac{1}{2} k y_{u}^{2}+\frac{1}{3} k_{2} y_{u}^{3}+\frac{1}{4} k_{3} y_{u}^{4}+\frac{V^{2}}{2}\left(C_{0}+C_{0} \frac{y_{u}}{y_{\mathrm{ov}}}\right)
\end{aligned}
$$

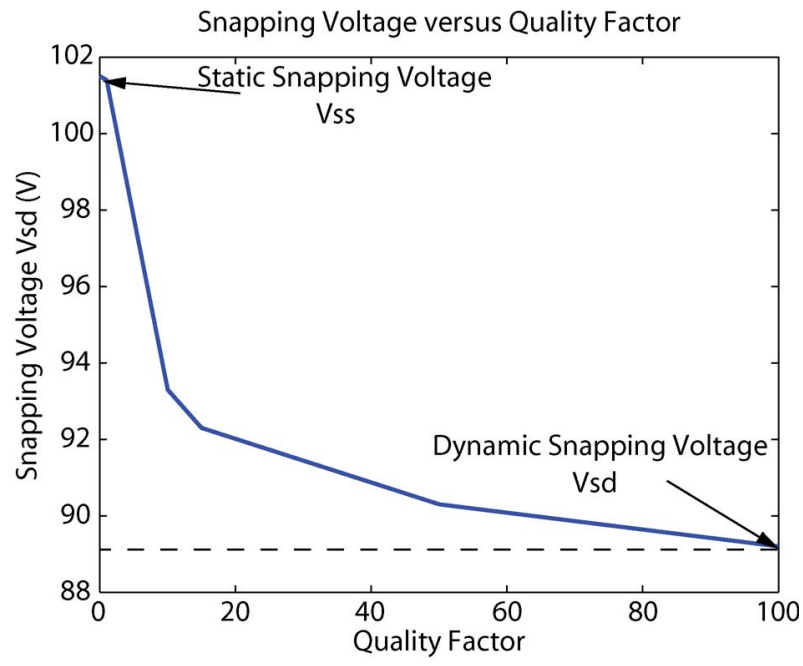

Fig. 6. Evolution of the snapping voltage as a function of the quality factor (static snapping voltage in this example $V_{\mathrm{ss}}=101.3 \mathrm{~V}$, dynamic snapping voltage $V_{\mathrm{sd}}=89.2 \mathrm{~V}$ ).

where the unstable points or critical points $\left(y_{u}\right)$ satisfy the conditions $d U / d y=0$ and $d U^{2} / d^{2} y<0$. The critical point $\left(y_{u}\right)$ and the dynamic snapping voltage can be calculated as

$$
\begin{aligned}
y_{u} & =-\frac{1}{9} \frac{4 k_{2}-\sqrt{16 k_{2}^{2}-54 k_{3} k}}{k_{3}} \\
V_{\mathrm{sd}} & =\frac{\sqrt{-2 N \varepsilon_{0} z_{0} y_{u} g_{0}\left(k_{3} y_{u}^{2}+k_{2} y_{u}+k\right)}}{N \varepsilon_{0} z_{0}} .
\end{aligned}
$$

If damping forces are introduced, the voltage calculated by (38) has to be increased, because the energy losses caused by the damping force prevent from overshooting the critical point. Fig. 6 shows the variation of the snapping voltage against the quality factor. It can be observed that the dynamic snapping voltage decreases $\left(V_{\mathrm{sd}}\right)$ from the static snapping voltage $\left(V_{\mathrm{ss}}\right)$, when the quality factor $Q$ is increased, until the dynamic snapping voltage is achieved for values of $Q$ larger than 100 .

When the system does not have enough energy to switch to the second state, the energy decreases until the first stable equilibrium point is reached. However, if a voltage larger than the dynamic snapping voltage is applied, the system has enough energy to switch to the second stable state. Fig. 7 shows that the dynamic snapping occurs as predicted by model, the system has the initial energy equal to the energy necessary to overcome the critical energy point (or maximum on the energy curve). Fig. 8 shows snapping to the second stable state of the bistable micromechanism when a step voltage of $92.4 \mathrm{~V}$ is applied, while the calculated static snapping voltage is $101.3 \mathrm{~V}$. Therefore, if the system is actuated dynamically, the switch to the second stable state can be achieved with a voltage of $11.9 \%$ lower than the static voltage depending on the value of $Q$. If the quality factor is increased, the value of voltage will subsequently decrease.

\section{Dynamic Snapping Due to Harmonic Forcing}

In the case of a linear system when driven with a force at the resonance frequency, the system achieves large amplitude of 


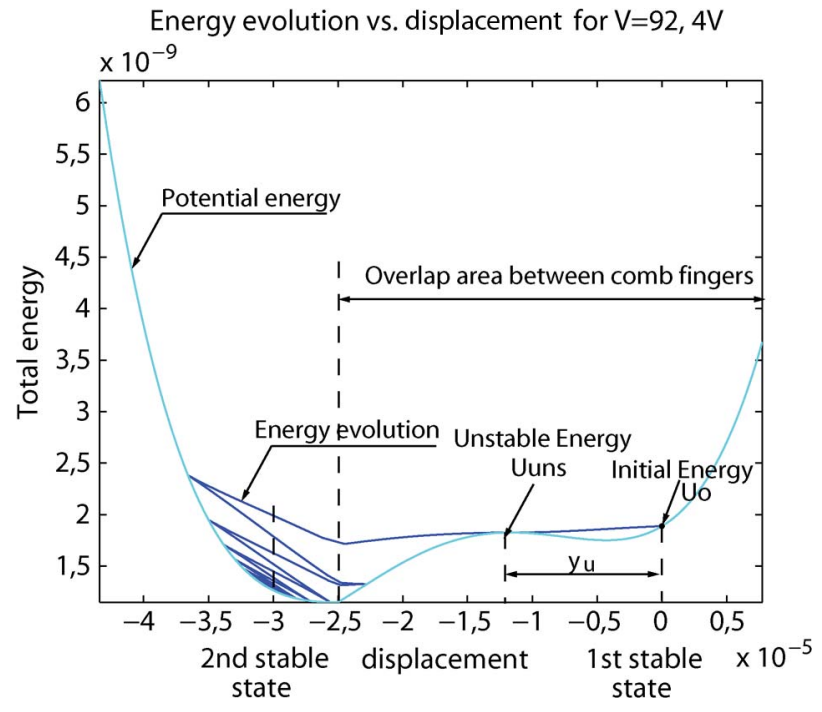

Fig. 7. Evolution of the total energy for a step input of $92.4 \mathrm{~V}$. The unstable condition is achieved, and the system switch to the second stable state where it oscillates until the energy is completely dissipated.

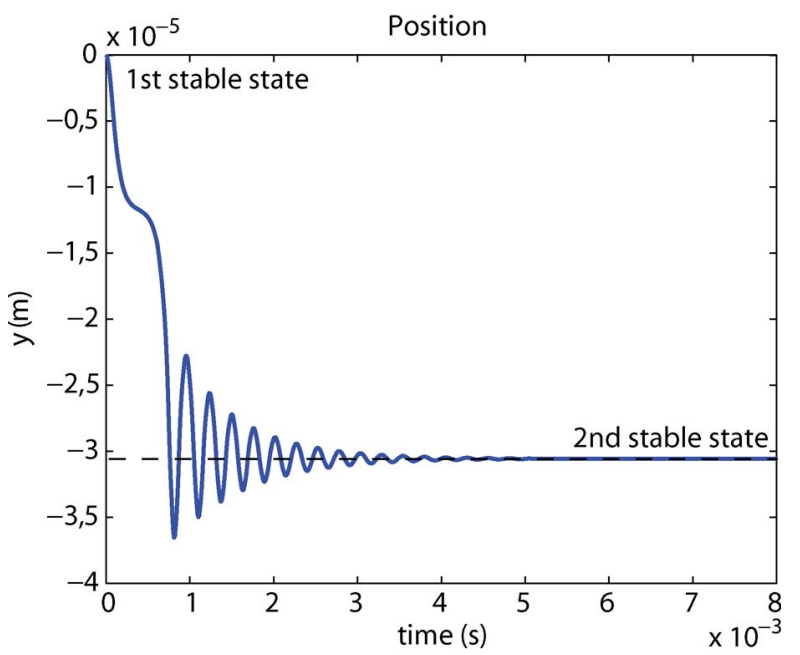

Fig. 8. Evolution of the position of the central point of the double built-in prebuckled beam when a voltage is applied following a step input of $92.4 \mathrm{~V}$ $(Q=10)$.

resonant oscillation. If this phenomena is applied to a nonlinear system, such as a bistable mechanism, a "resonantlike" large oscillations may provide enough deviation from the first stable state to switch to the second stable state. In this case, the snapping can occur at relatively low voltages.

The study of the energy is presented for the case of forced oscillation. The voltage applied through the comb-drive actuation is $V(t)=V_{\mathrm{dc}}+v_{\mathrm{ac}}(t)$, where $V_{\mathrm{dc}}$ is a constant voltage and $v_{\mathrm{ac}}(t)$ is a square-wave signal. The equilibrium points or zeros of the variation of the energy depend on the actuation voltage

$$
\frac{d U}{d y}=k y+k_{2} y^{2}+k_{3} y^{3}+\frac{V(t)^{2}}{2}\left(C_{0} \frac{1}{y_{\mathrm{ov}}}\right)=0
$$

Equation (39) implies that energy is changing continuously, since $V(t)$ will change between $|V|=\left|V_{\mathrm{dc}}\right|-\left|v_{\mathrm{ac}}\right|$ and $|V|=$ $\left|V_{\mathrm{dc}}\right|+\left|v_{\mathrm{ac}}\right|$ every half cycle. Due to dynamics of the system,

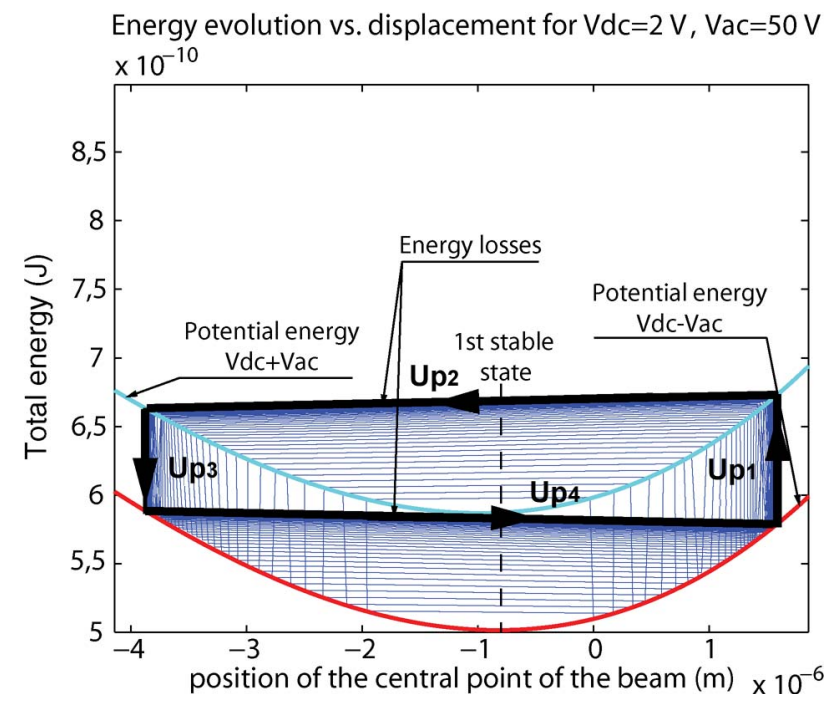

Fig. 9. Evolution of the position of a double built-in prebuckled beam. Voltages applied $V_{\mathrm{dc}}=2 \mathrm{~V}$ and $V_{\mathrm{ac}}=50 \mathrm{~V}(Q=30)$.

the damping and the inertia has to be considered [(34)]. In steady-state oscillation, an energy is exchanged between the energy provided to the system due to harmonic oscillation $\left(U_{p 1}\right.$ and $\left.U_{p 3}\right)$ and the energy lost due to damping ( $U_{p 2}$ and $U_{p 4}$ ). In Fig. 9, it is shown that, at a stable resonant frequency, there is an amplitude of oscillation where the energy balance is zero [15]

$$
U_{p 1}+U_{p 2}+U_{p 3}+U_{p 4}=0
$$

where $U_{p 1}$ and $U_{p 3}$ can be analytically calculated as the difference between the potential energy curves.

$$
\begin{aligned}
U_{p 1}= & U\left(V(t)=V_{\mathrm{dc}}+v_{\mathrm{ac}}(t)\right)-U\left(V(t)=V_{\mathrm{dc}}-v_{\mathrm{ac}}(t)\right) \\
= & \frac{1}{2}\left(C_{0}+\frac{N \varepsilon_{o} z_{0} y_{u \min }}{g_{0}}\right) \\
& \times\left(\left(V_{\mathrm{dc}}+v_{\mathrm{ac}}(t)\right)^{2}-\left(V_{\mathrm{dc}}-v_{\mathrm{ac}}(t)\right)^{2}\right) \\
U_{p 3}= & U\left(V(t)=V_{\mathrm{dc}}-v_{\mathrm{ac}}(t)\right)-U\left(V(t)=V_{\mathrm{dc}}+v_{\mathrm{ac}}(t)\right) \\
= & \frac{1}{2}\left(C_{0}+\frac{N \varepsilon_{o} z_{0}\left(-y_{u \min }\right)}{g_{0}}\right) \\
& \times\left(\left(V_{\mathrm{dc}}-v_{\mathrm{ac}}(t)\right)^{2}-\left(V_{\mathrm{dc}}+v_{\mathrm{ac}}(t)\right)^{2}\right) .
\end{aligned}
$$

In (40), $U_{p 2}$ and $U_{p 4}$ are the energy losses due to damping during $|V|=\left|V_{\mathrm{dc}}\right|-\left|v_{\mathrm{ac}}\right|$ and $|V|=\left|V_{\mathrm{dc}}\right|+\left|v_{\mathrm{ac}}\right|$ half periods, respectively. At resonance, if the motion of the beam is considered approximately sinusoidal, the energy dissipated by the viscous damping effect in one half cycle is

$$
U_{p 2}=U_{p 4}=-\frac{\pi}{2} \omega B y_{u \min }^{2}
$$

where $y_{u \text { min }}$ is minimum deviation from the first stable equilibrium that produces snapping to the second stable state. When there is enough energy stored in the system, the amplitude of oscillation overcomes the critical point. Fig. 10 shows the evolution of the total energy in the system which is bounded 


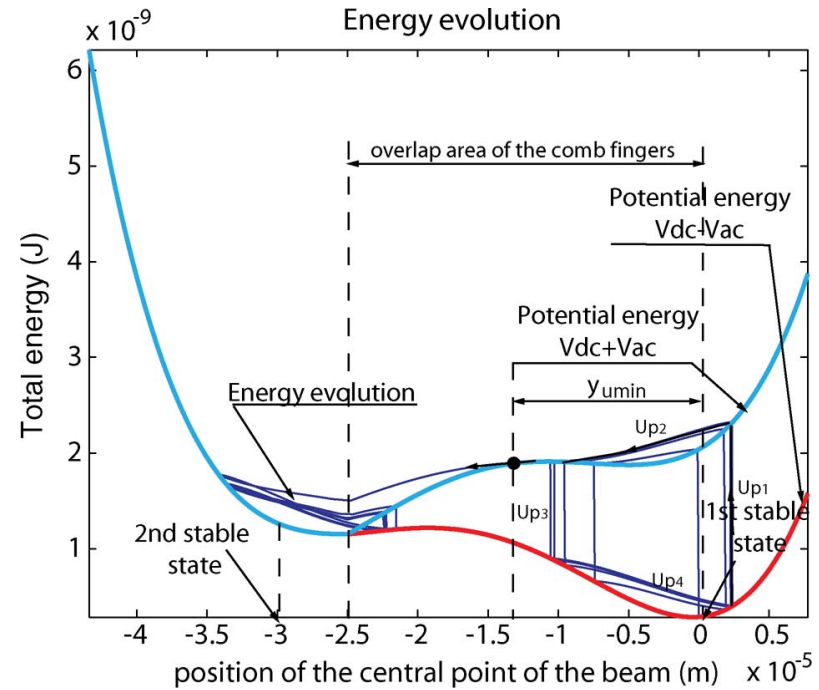

Fig. 10. Evolution of the position of a double built-in prebuckled beam. Voltages applied $V_{\mathrm{dc}}=2 \mathrm{~V}$ and $V_{\mathrm{ac}}=80 \mathrm{~V}(Q=30)$.

by the corresponding potential energy curves; the first curve corresponds to the case when both voltages are added $(|V|=$ $\left.\left|V_{\mathrm{dc}}\right|+\left|v_{\mathrm{ac}}\right|\right)$ and the second curve when the voltages are subtracted $\left(|V|=\left|V_{\mathrm{dc}}\right|-\left|v_{\mathrm{ac}}\right|\right)$. Consequently, the oscillation amplitude that has to be achieved to switch to the second stable state is defined by the maximum potential energy $(|V|=$ $\left.\left|V_{\mathrm{dc}}\right|+\left|v_{\mathrm{ac}}\right|\right)$, called $y_{u \text { min }}$

$$
\begin{aligned}
k y_{u \text { min }}+k_{2} y_{u \min }^{2}+ & k_{3} y_{u \text { min }}^{3} \\
& +\frac{\left(\left|V_{\mathrm{dc}}\right|+\left|v_{\mathrm{ac}}\right|\right)^{2}}{2}\left(C_{0} \frac{1}{y_{\mathrm{ov}}}\right)=0 .
\end{aligned}
$$

Substituting (41)-(43) in (40), yields to the combination of voltages, $V_{\mathrm{dc}}$ plus a $v_{\mathrm{ac}}(t)$ square wave signal that applied simultaneously will produce snapping to the second bistable state. The ac dynamic snapping voltage $V_{\mathrm{ACDS}}$ is the combination of minimum $\mathrm{dc}$ and ac voltages producing snapping

$$
V_{\mathrm{ACDS}}=v_{\mathrm{ac}} \times V_{\mathrm{dc}}=\frac{B y_{u \min } \omega \pi g_{0}}{2 N \varepsilon 0 z_{0}} .
$$

In (44), the parameter $B$ is the damping coefficient, $\omega$ is the frequency of the driving voltage $v_{\mathrm{ac}}, y_{u \text { min }}$ is the minimum amplitude that causes snapping, $N$ is the number of pairs of comb-drive plates, $g_{0}$ is the distance between fingers, $z_{0}$ is the thickness of the comb drives, and $\varepsilon_{0}$ is the dielectric permittivity.

The same analysis has been done using the governing equation [(23)]. For illustration, we use the dimensions of the fabricated devices, which are summarized in Table II. Fig. 11 shows influence of the quality factor $Q$ on the voltages needed to achieve the second stable state, which means that, for instance, the voltage required can be modified by packaging these type of devices at moderate vacuum.

The modeling illustrates that, for low ac actuation voltages $(\sim 0.5 \mathrm{~V})$, the $V_{\mathrm{dc}}$ required to switch between stable states is almost constant despite variation in quality factor $(Q)$. In this case, the system behaves as if the input were a step function, and the dynamic advantage is due to dynamic overshoot when

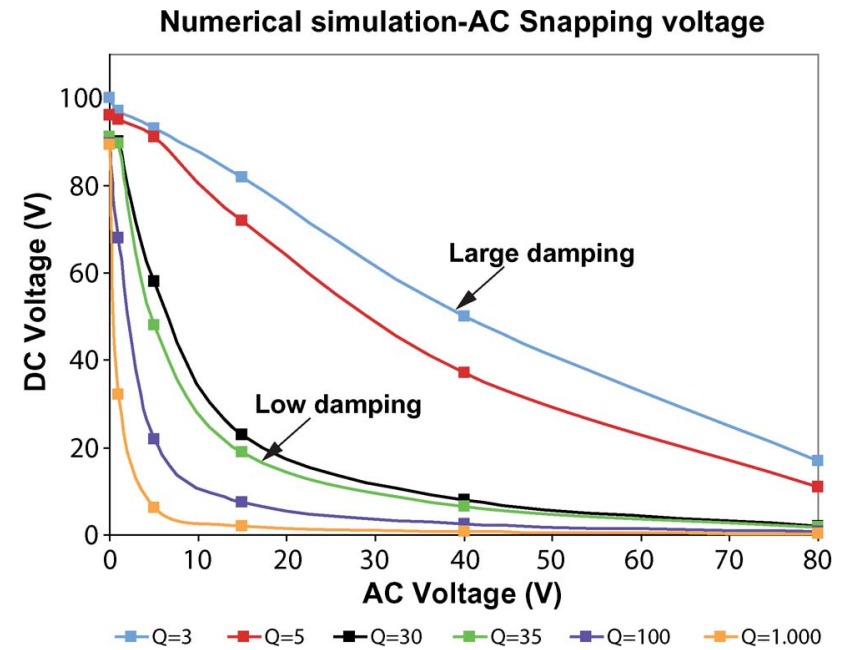

Fig. 11. Numerical simulations of the ac dynamic snapping voltage for a fixed quality factor $Q$. The plot represents minimum dc and ac voltages applied simultaneously to produce switching between stable states.

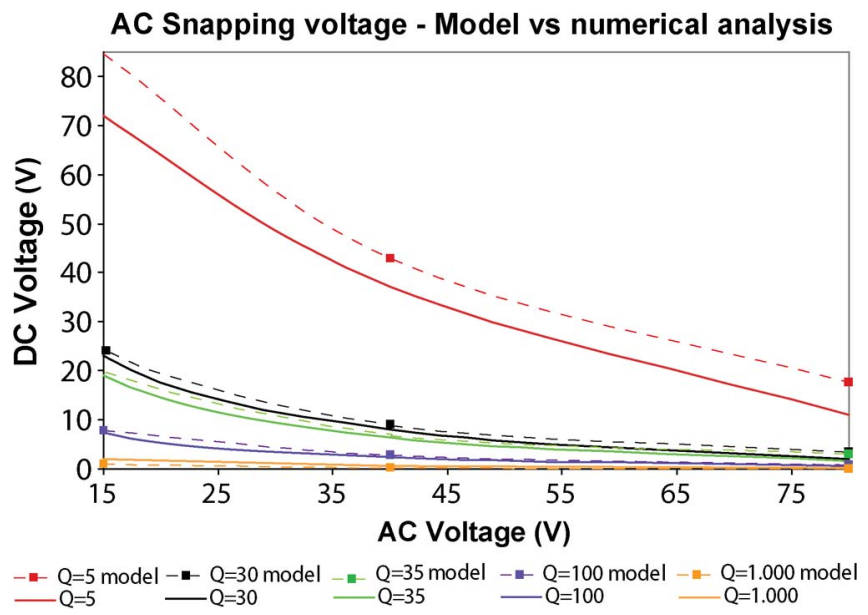

Fig. 12. Comparison between the ac dynamic snapping voltage calculated (continuous) using numerical simulations and (dashed) using the analytical model.

studying $V_{\text {sd }}$ dynamic voltage. When ac voltages are increased and if the quality factor is low, there is a significant loss of energy due to damping. The relationship between both voltages follows a linear trend, and the required snapping voltages are similar in both cases (large $V_{\mathrm{dc}}+$ low $V_{\mathrm{ac}}$ or low $V_{\mathrm{dc}}+$ large $V_{\mathrm{ac}}$ ). If $Q$ is increased, the relationship between $V_{\mathrm{dc}}$ and $V_{\text {ac }}$ is hyperbolic, causing a decrease of the needed voltages to achieve snapping. This property offers the possibility to achieve snapping with less voltage applied than if the actuation were only a constant voltage (pull-in voltage, $V_{\mathrm{ss}}=101.3 \mathrm{~V}$ ). The relationship between both voltages has been analytically derived in (44).

Fig. 12 shows a comparison of the predicted combination of voltages needed for switching using the analytical expressions and the numerical model. From (44), the influence of the quality factor $Q$ on the voltages needed to achieve the second stable state is evident. The relation between $V_{\mathrm{dc}}$ and $v_{\mathrm{ac}}$ is hyperbolic; this behavior can be also appreciated in the numerical simulations shown in Fig. 11. The analytical expression provides a first approximation of voltages needed without timeconsuming numerical simulations. For the devices analyzed, 
TABLE I

Average Increase Between Voltages NeEded Using (DASHed) THE ANAlytical MODEL AND (CONTINUOUS) THE NumeriCAL Simulations. SEE Fig. 12

\begin{tabular}{|c|c|c|c|c|}
\hline $\mathrm{Q}=5$ & $\mathrm{Q}=30$ & $\mathrm{Q}=35$ & $\mathrm{Q}=100$ & $\mathrm{Q}=1000$ \\
\hline $20,70 \%$ & $13,88 \%$ & $15,31 \%$ & $12,63 \%$ & $12,50 \%$ \\
\hline
\end{tabular}

the average error is $15 \%$, but the error increases for low values of quality factor and doubles to around $30 \%$, see Table I. In the low- $Q$ domain, the effect of the kinetic energy is rapidly compensated by the importance of the loss of energy. In this case, the relation between $V_{\mathrm{dc}}$ and $V_{\mathrm{ac}}$ tends to be more linear. In this case, the second stable state is only achieved if, due to the initial overshoot, the critical energy is over passed.

The domain of validity of the presented model of the dynamics in describing the snapping behavior can be summarized as follows.

1) If a low ac voltage is applied, the $V_{\mathrm{dc}}$ necessary to change between stable states is almost constant. In this case, the force applied is almost constant, and the dynamic parameters of the system do not play an important role. The system behaves as if the input were a step function, and the best applied voltage in this case is $V_{\text {sd }}$ defined by (38).

2) For quality factors lower than ten, when there is loss of energy due to damping, the required snapping voltages are similar in both cases (large $V_{\mathrm{dc}}+$ low $V_{\mathrm{ac}}$ or low $V_{\mathrm{dc}}+$ large $V$ ac), see Fig. 11. In this case, the analytical expression overestimates the voltages needed and is around $\sim 30 \%$.

3) For quality factors higher than ten, the relationship between $V_{\mathrm{dc}}$ and $V_{\mathrm{ac}}$ is hyperbolic, causing a decrease of the needed voltages to achieve snapping. The relationship between both voltages has been analytically derived in (44), and it accurately predicts the needed voltages with errors lower than 15\%, see Fig. 12.

\section{EXPERIMENTAL VALIDATION}

SOI prototypes of bistable vibratory devices were fabricated in order to experimentally validate dynamic snapping advantages in terms of power saving as compared to static snapping. The structures were fabricated using the one-mask bulkmicromachining process, based on deep reactive-ion etching through a 50- $\mu \mathrm{m}$-thick silicon layer of SOI wafers. The structures were released by removing the oxide layer using aqueous hydrofluoric acid. Not removed oxide serves as an anchor. In Table II, dimensions of the devices used for experiments are summarized. These parameters have been obtained based on a previously discussed optimization using the analytical model. The parameters were corrected by taking into account fabrication imperfections observed in previous fabrication runs. Fig. 1 shows the main parts of the prototypes: two separate built-in buckled beams, the contact of the second stable state, and the actuation based on comb-drive fingers.

Our experiments included the following procedures: detection of the resonant frequency and experimental determination of the quality factor $(Q)$ and evaluation of the combination of $V_{\mathrm{dc}}+v_{\mathrm{ac}}$ voltages that cause switching between bistable states.
TABLE II

Dimensions of the FABRICATEd BUILT-In PREBUCKLED BISTABLE MICROSWITCH

\begin{tabular}{|c|c|c|}
\hline Dimensions & Description & Value \\
\hline $\mathrm{L}$ & Beam length & $3.000 \mu \mathrm{m}$ \\
\hline $\mathrm{W}$ & Beam width & $6,5 \mu \mathrm{m}$ \\
\hline$z_{0}$ & Thickness & $50 \mu \mathrm{m}$ \\
\hline$b$ & Initial buckling & $15,5 \mu \mathrm{m}$ \\
\hline $\mathrm{N}$ & Number of combs & 200 \\
\hline$y_{o v}$ & Overlap between combs & $25 \mu \mathrm{m}$ \\
\hline$g_{0}$ & Gap between combs & $7 \mu \mathrm{m}$ \\
\hline
\end{tabular}

When the device is driven at resonance, the motion generated is detected electronically by extracting the motion-induced current. The electronic circuit is formed by a dc power source, model Agilent E3631A, to provide the bias voltage, ac power source (internal oscillator of the DSP 7260 Lock-In amplifier) to provide the ac voltage, two dc sources to power the transimpedance amplifier (model Lascar PSU 130), a transimpedance amplifier circuit (based on LM837 amplifier), a dynamic signal analyzer (Signal Recovery DSP 7260 Lock-In amplifier), a vacuum chamber (fabricated in-house), and a vacuum pump (model Leybold trivac E2).

The current induced by motional response is electronically detected using a transimpedance amplifier-based circuit [24]. The lock-in amplifier DSP 7260 measures signal at the first harmonic, filtering the fundament frequency and eliminating parasitics. Fig. 13 shows swept sine measurements reading first harmonic at atmospheric pressure. The peak increases with an increase of $v_{\text {ac }}$, see Fig. 13(b), which is expected as the output voltage is proportional to the reference $v_{\mathrm{ac}}$ voltage amplitude and the amplitude of oscillation. The frequency response using first harmonic shows better signal-tonoise ratio, as the frequency of excitation is separated from the mechanical-oscillation frequency response. The amplitude increase is quadratically dependent on $v_{\mathrm{ac}}$ voltage and linearly dependent on $V_{\mathrm{dc}}$ voltage. The same swept sine measurements were done under vacuum, see Fig. 14, showing a sharper resonance region due to less viscous damping. All these measurements were done with low voltages applied due to limitation of voltage source in the lock-in amplifier. The responses obtained were small-amplitude oscillation around the equilibrium position in the linear region of the behavior of the device. Measurements at atmospheric pressure showed a broader frequency response. In Fig. 14(b), a small shift in frequency due to the change in the initial equilibrium position due to the resulting force balance is observed, caused by the increasing dc voltage applied. From the first harmonic response, an accurate extraction of the resonance frequency has been done (ranging between 4063 and $4093 \mathrm{~Hz}$ ). The resonance frequency of the prototype agrees with the expected frequency obtained by FEA $(4018 \mathrm{~Hz})$. A reasonable mismatch of up to $3 \%$ is expected due to measurement errors and fabrication imperfections.

Using the $-3-\mathrm{dB}$ bandwidth-detection method, the quality factor of the system was determined from the frequencyresponse curve, see Table III. The prototypes were driven using different combinations of $V_{\mathrm{dc}}+v_{\mathrm{ac}}$ voltages dynamically to demonstrated the switching between stable states at lower voltages as compared to static voltage. The dc power supply and 


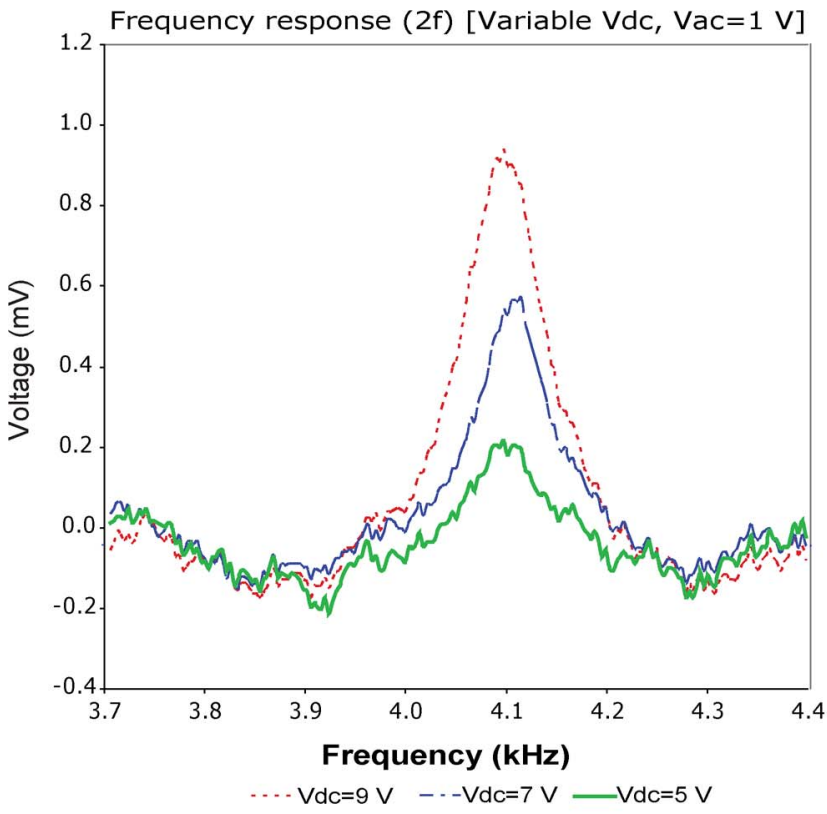

(a)

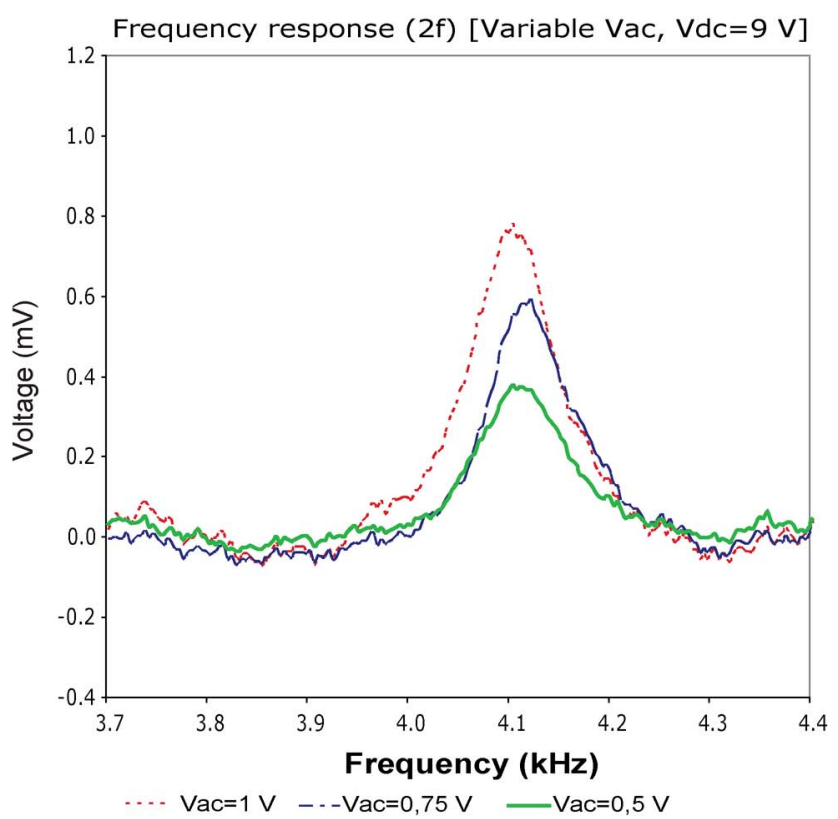

(b)

Fig. 13. Swept sine measurements at atmospheric pressure. (a) Frequency response of first-harmonic frequency (2f) with variable $V_{\mathrm{dc}}$ ranging from 3 to $9 \mathrm{~V}\left(v_{\mathrm{ac}}=1 \mathrm{~V}\right)$. (b) Frequency response of first-harmonic frequency (2f) with peak-to-peak amplitudes changing from 1 to $0.5 \mathrm{~V}$ and constant dc voltage $\left(V_{\mathrm{dc}}=9 \mathrm{~V}\right)$.

the ac function generator are used to provide the combination of voltages needed to cause snapping at resonance frequency. Experimental tests of dynamic switching have been carried out to extract the ac dynamic snapping voltages. The response is detected optically through a $\mathrm{C}$-mount microscope video camera "Pixel Link PL-A662" illuminated utilizing fiber-optic illumination "Fiber lite PL-900," which is connected to the PC through a firewire port, allowing to visually monitor response of the device. While the device is oscillating, the $v_{\mathrm{ac}}$ is fixed, and the $V_{\mathrm{dc}}$ is increasing until the switching to the second state occurs.

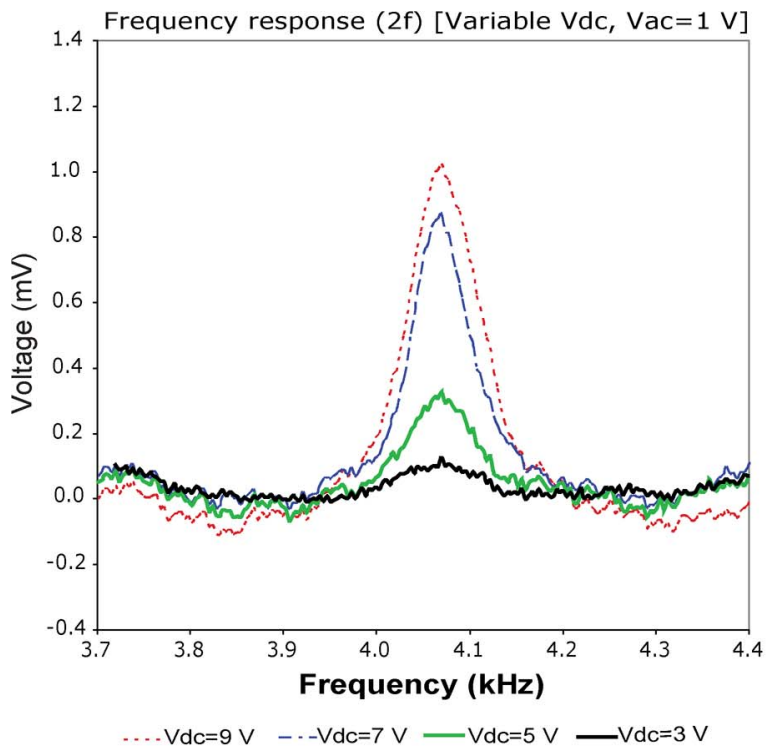

(a)

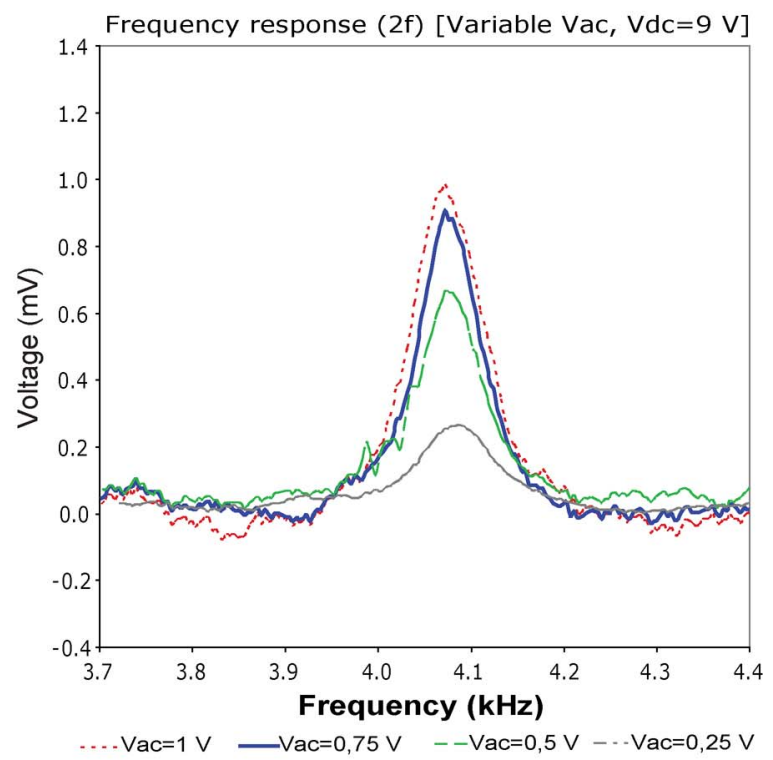

(b)

Fig. 14. Swept sine measurements at 75 torr. (a) Frequency response of firstharmonic frequency (2f) width variable $V$ dc ranging from 3 to $9 \mathrm{~V}\left(v_{\mathrm{ac}}=\right.$ $1 \mathrm{~V}$ ). (b) Frequency response of first-harmonic frequency (2f) with variable with peak-to-peak amplitudes changing from 1 to $0.5 \mathrm{~V}$ and constant dc voltage $\left(V_{\mathrm{dc}}=9 \mathrm{~V}\right)$.

TABLE III

EXPERIMENTALLy Measured AVERAge Quality FaCtor $(\bar{Q})$

\begin{tabular}{|c|c|}
\hline Pressure & Q-factor \\
\hline Vacuum (75 Torr) & 35,5 \\
Atmospheric Pressure (760 Torr) & 29,9 \\
\hline
\end{tabular}

Fig. 15 shows the combination of ac and dc voltages required to produce the dynamic switching between stable states. As it can be observed, the analytical model predicts accurately the combination of voltages that produce dynamic switching between states. The results depend on the damping of the system (quality factor, $Q$ ). The test was performed in air $(P=$ 760 torr, $\bar{Q}=29.9)$ and at reduced pressure $(P=75$ torr, $\bar{Q}=35.5)$. Based on analytical predictions, the static snapping voltage, for the dimensions of the prototype, is around $101.3 \mathrm{~V}$. 


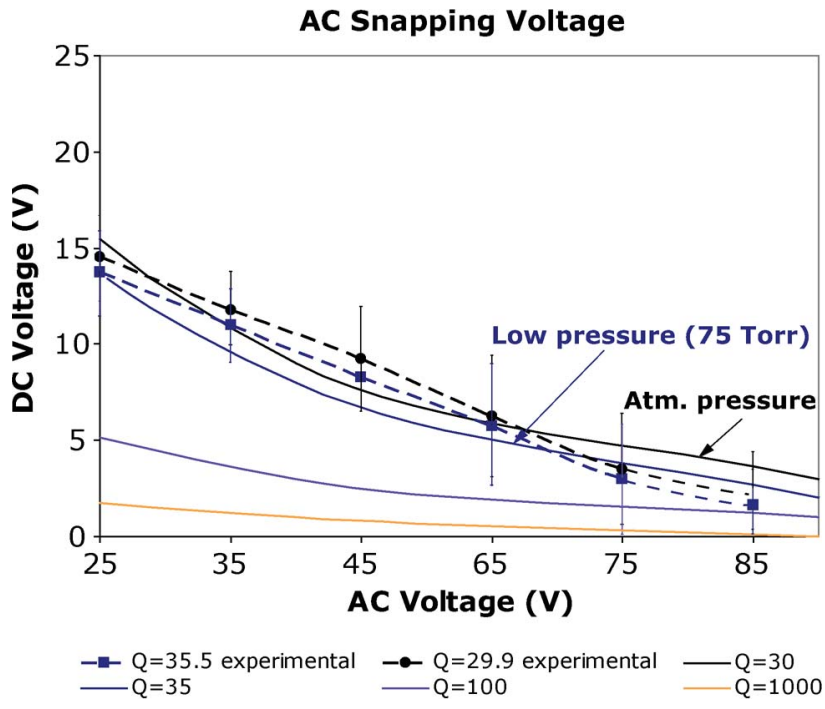

Fig. 15. Comparison between the numerical simulation and the experimental values for which the built-in prebuckled beams achieve ac dynamic snapping. (a) AC dynamic snapping at 760 torr. (b) AC dynamic snapping at 75 torr.

At atmospheric pressure, maximum efficiency is achieved when $V_{\mathrm{dc}}=14 \mathrm{~V}$ and $v_{\mathrm{ac}}=25 \mathrm{~V}$, with a voltage reduction compared to static snapping of $62.5 \%$. This verifies that dynamic switching between states requires lower voltages. The decrease of the ambient pressure to $10 \%$ improves the voltage savings up to $66.4 \%$. The obtained results confirm that the model is accurate enough to predict the phenomena of dynamic switching of bistable structures.

\section{CONCLUSION}

In this paper, the design, analysis, fabrication, and experimental verification of a dynamic switching strategy for bistable snap-action mechanisms have been presented. This is the first bistable-mechanism design which suggests to take advantage of "resonancelike" phenomena in bistable mechanisms to switch between stable states. The complete dynamic model using energy analysis of a bistable built-in prebuckled beam has been developed to validate the advantage of driving bistable devices using the "resonancelike" phenomena in bistable mechanisms. The combination of ac and dc voltages needed to produce dynamic snapping has been estimated using an analytical model and compared to experimental results, demonstrating an error lower than $15 \%$ for actuation under 75 torr (Fig. 15). This model has demonstrated to be well suited to model high- $Q$ situations, but for low- $Q$ domain where the kinetic energy is rapidly compensated by the energy losses due to damping, the relation between both voltages has a linear trend and summation of both voltages has to be similar to $V_{\text {sd }}$ (dynamic snapping voltage). Further analysis needs to be done to incorporate more accurate models of the damping in the model. The effect of material/intrinsic damping should be analyzed, and the effect of the damping forces over the velocity (dynamics of the systems) should be carefully studied. To verify the dependence of actuation on the quality factor $Q$, two sets of experiments have been conducted in air ( $\bar{Q}=29.9, P=760$ torr $)$ and at low pressure $(\bar{Q}=35.5, P=75$ torr $)$. The static snapping voltage was estimated to be around 101.3 V. When resonance actuation was used, the combination of voltages needed to switch between states was reduced by $62.5 \%$ at atmospheric pressure $(Q=29.9)$ and up to $66.4 \%$ at 75 torr $(Q=35.5)$. This demonstrates the advantages of dynamic switching, allowing the switching between states at lower voltages.

\section{REFERENCES}

[1] M. S. Baker, S. M. Lyon, and L. L. Howell, "A linear displacement bistable micromechanism," in Proc. ASME Des. Eng. Tech. Conf. DETC2000/MECH-14117, 2000, pp. 1-7.

[2] J. Qiu, J. H. Lang, and A. H. Slocum, "A centrally-clamped parallel-beam bistable MEMS mechanism," in Proc. IEEE MEMS, 2001, pp. 353-356.

[3] M. Hoffmann, P. Kopka, and E. Voges, "All-silicon bistable micromechanical fiber switch based on advanced bulk micromachining," IEEE J. Sel. Topics Quantum Electron., vol. 5, no. 1, pp. 46-51, Jan./Feb. 1999.

[4] E. J. Kruglick and S. J. Pister, "Bistable MEMS relays and contact characterization," in Proc. IEEE Solid-State Sens. Actuator Workshop, Jun. 1998, pp. 333-337.

[5] B. D. Jensen, "Identification of macro- and micro-compliant mechanism configurations resulting in bistable behavior," M.S. thesis, Brigham Young Univ., Provo, UT, 1998.

[6] M. T. A. Saif, "On a tunable bistable MEMS - Theory and experiment," J. Microelectromech. Syst., vol. 9, no. 2, pp. 157-170, Jun. 2000.

[7] B. Halg, "On a nonvolatile memory cell based on micro-electromechanics," in Proc. IEEE Micro Electro Mech. Syst., 1990, pp. 172-176.

[8] L. Buchaillot, O. Millet, E. Quévy, and D. Collard, "Post-Buckling dynamic behavior of self-assembled 3D microstructures," Microsyst. Technol., vol. 14, no. 1, pp. 69-78, Aug. 2007.

[9] A. H. Nayfeh and M. I. Younis, "A new approach to the modeling and simulation of flexible microstructures under the effect of squeeze-film damping," J. Micromech. Microeng., vol. 14, no. 2, pp. 170-181, Feb. 2004.

[10] Y.-S. Shim, I.-H. Hwang, and J.-H. Lee, "Modeling and experimental characterization of the Chevron-type bi-stable microactuator," J. Micromech. Microeng., vol. 13, no. 6, pp. 948-954, Nov. 2003.

[11] J. S. Go, Y.-H. Cho, B. M. Kwak, and K. Park, "Snapping microswitches with adjustable acceleration threshold," Sens. Actuators, vol. A54, no. 1-3, pp. 579-583, Jun. 1996.

[12] C. Nguyen, "Frequency-selective MEMS for miniaturized low-power communication devices," IEEE Trans. Microw. Theory Tech., vol. 47, no. 8, pp. 1486-1503, Aug. 1999.

[13] V. Milanovic, M. Maharbiz, A. Singh, B. Warneke, N. Zhou, H. K. Chan, and K. S. Pister, "Microrelays for batch transfer integration in RF systems," in Proc. IEEE Int. Conf. Microelectromech. Syst., Tokyo, Japan, 2000, pp. 787-792.

[14] G. M. Rebeiz and J. B. Muldavin, "RF MEMS switches and switch circuits," IEEE Microw. Mag., vol. 2, no. 4, pp. 59-71, Dec. 2001.

[15] A. Fargas-Marques, J. Casals-Terre, and A. Shkel, "Resonant pull-in condition in parallel-plate electrostatic actuators," J. Microelectromech. Syst., vol. 16, no. 5, pp. 1044-1053, Oct. 2007.

[16] S. S. Rao, Mechanical Vibrations, 2nd ed. Reading, MA: AddisonWesley, 1990.

[17] C. M. Wang, C. Y. Wang, and J. N. Reddy, Exact Solutions for Buckling of Structural Members. Boca Raton, FL: CRC Press, 2004.

[18] G. J. Simitses, Dynamic Stability of Suddenly Loaded Structures. New York: Springer-Verlag, 1999.

[19] W. Lacarbonara, "A theoretical and experimental investigation of nonlinear vibrations of buckled beams," M.S. thesis, Virginia Polytechnic Inst. State Univ., Blacksburg, VA, 1997.

[20] W. Lacarbonara, A. H. Nayfeh, and W. Kreider, "Experimental validation of reduction methods for nonlinear vibrations of distributed-parameter systems: Analysis of a buckled beam," Nonlinear Dyn., vol. 17, no. 2, pp. 95-117, Oct. 1998.

[21] S. A. Emam, "A theoretical and experimental study of nonlinear dynamics of buckled beams," Ph.D. dissertation, Virginia Polytechnic Inst. State Univ., Blacksburg, VA, 2002.

[22] W. C. Tang, T. H. Nguyen, and R. T. Howe, "Laterally driven polysilicon resonant microstructures," Sens. Actuators, vol. 20, no. $1 / 2$, pp. 25-32, Nov. 1989.

[23] T. A. Roessig, "Integrated MEMS tuning fork oscillators for sensor applications," Ph.D. dissertation, Univ. California, Berkeley, CA, 1998.

[24] C. H. Nguyen, "Electromechanical characterization of microresonators for circuit applications," M.S. thesis, Univ. California, Berkeley, CA, 1991. 


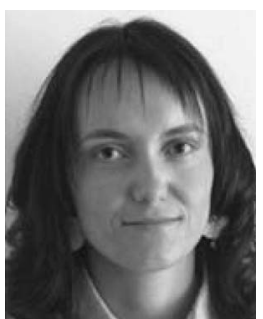

Jasmina Casals-Terré received the B.S. degree in mechanical engineering from the Technical University of Catalonia (UPC), Terrassa, Spain, in 2000, the M.S. degree in mechanical and aerospace engineering from the University of California, Irvine, Irvine, in 2002, and the Ph.D. degree in mechanical engineering from UPC in 2007.

Since 2003, she has been a member of the Faculty of UPC, where she is currently a Lecturer in the Department of Mechanical Engineering. Her research interests include design, fabrication, and experimental study of nonlinear phenomena in dynamic microelectromechanical systems.

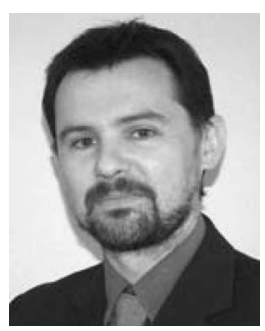

Andreu Fargas-Marques received the Diploma degree (with excellence) in industrial engineering from the Technical University of Catalonia (UPC), Barcelona, Spain, in 2000, and the M.S. degree in mechanical and aerospace engineering from the University of California, Irvine, Irvine, in 2001. He is currently working toward the Ph.D. degree in advanced control of microelectromechanical systems (MEMS) in the Institute of Industrial and Control Engineering, UPC

He was a Project Engineer with SENER Engineering. His research interests include the control of MEMS, particularly those actuated electrostatically such as resonators, switches, or gyroscopes.

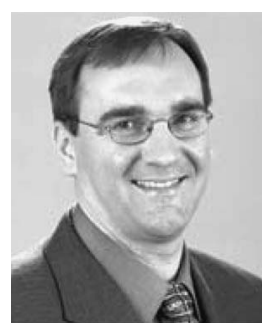

Andrei M. Shkel (S'95-A'98) received the Diploma degree (with excellence) in mechanics and mathematics from Moscow State University, Moscow, Russia, in 1991, and the Ph.D. degree in mechanical engineering from the University of Wisconsin, Madison, in 1997.

He was a Postdoctoral Researcher with the Berkeley Sensor and Actuator Center. He then held research and consulting positions with several hightech and venture companies. Since 2000, he has been a member of the faculty of the University of California, Irvine (UCI), where he is currently an Associate Professor in the Department of Mechanical and Aerospace Engineering, Department of Electrical Engineering and Computer Sciences, and Department of Biomedical Engineering. $\mathrm{He}$ is also the Director of the UCI Microsystems Laboratory. $\mathrm{He}$ is the holder of ten U.S. and international patents (with 13 pending) on micromachined angle-measuring gyroscopes, wide-bandwidth rate gyroscopes, design and fabrication of light manipulators and tunable optical filters, and hybrid micromachining processes. His professional interests, which are reflected in more than 100 publications, include solid-state sensors and actuators, microelectromechanical-system-based neuroprosthetics, sensor-based intelligence, and control theory.

Dr. Shkel is an Editor of the Journal of Microelectromechanical SYsTEMS. He was a Guest Editor for two Special Issues of the IEEE SENSORS Journal. He was the General Chair of the 2005 IEEE Sensors Conference and the Vice General Chair and Publications Chair of the 2002-2006 IEEE Sensors Conferences. He is currently the Vice President of Conferences of the IEEE Sensors Council. He was the recipient of the 2006 Research Award of the UCI School of Engineering, the 2005 National Science Foundation CAREER Award, the 2002 George E. Brown, Jr., Award, and a 2001 Fellowship from the Japanese Advanced Science Institute. 